\title{
A case of refractory thrombotic thrombocytopenic purpura treated with plasmapheresis and rituximab
}

\author{
N Kirui, ${ }^{1,2} \mathrm{MB} \mathrm{ChB}$, MMed; A Sokwala, ${ }^{2} \mathrm{MD}$, MMed \\ ${ }^{1}$ Department of Internal Medicine, School of Medicine, College of Health Sciences, Moi University, Eldoret, Kenya \\ ${ }^{2}$ Aga Khan University Hospital, Nairobi, Kenya
}

Corresponding author: A Sokwala (drsokwala@gmail.com)

Thrombotic thrombocytopenic purpura (TTP) is a rare, life-threatening disorder with no prevalence or incidence studies in sub-Saharan Africa. Acquired TTP has several causes, all of which lead to decreased activity of von Willebrand factor cleaving protease (ADAMTS13) due to autoantibodies that are directed towards ADAMTS13. We report a case of a 46-year-old man who presented with most of the classic clinical manifestations of TTP.

S Afr Med J 2016;106(7):689-691. DOI:10.7196/SAMJ.2016.v106i7.9856

\section{Background}

Thrombotic thrombocytopenic purpura (TTP) is a rare, life-threatening disorder that occurs due to deficiency of ADAMTS13 (a disintegrin and metalloproteinase with a thrombospondin type 1 motif, member 13), which is a von Willebrand factor (VWF) cleaving protein. ${ }^{[1]}$

The absent or severely reduced activity of ADAMTS13 in patients with TTP prevents timely cleavage of unusually large multimers of VWF as they are secreted by endothelial cells, resulting in adhesion and aggregation of platelets in flowing blood, with formation of microvascular thrombi, which are responsible for most of the clinical features. ${ }^{[2]}$ Both congenital and acquired forms of TTP have been described. There are no prevalence or incidence studies on TTP in sub-Saharan Africa (SSA), but several case reports are available in literature. Scully et al. ${ }^{[3]}$ in the UK reported a TTP incidence rate of six cases per million people in 2008.

There are several causes of acquired TTP, all of which lead to decreased activity of ADAMTS13 due to autoantibodies that are directed towards ADAMTS13. ${ }^{[4]}$ The classic presentation of TTP is that which follows postinfectious intestinal infection with either shigatoxin-producing Escherichia coli or Shigella dysenteriae ${ }^{[5]}$ HIV infection has been directly associated with $\mathrm{TTP}^{[6]}$ and a case series by Gunther et al., ${ }^{[7]}$ in South Africa in 2007, demonstrated that HIVassociated TTP was the most common form of TTP. Postinfectious TTP is therefore the most common cause of TTP in SSA, where there is still a high incidence of infectious diseases.

TTP was originally characterised by a pentad of thrombocytopenia, microangiopathic haemolytic anaemia, fluctuating neurological deficits, renal impairment and fever, often with insidious onset. ${ }^{[8]}$ However, TTP can present without the full pentad. Up to $35 \%$ of patients do not have neurological signs at presentation, and renal abnormalities and fever are not always prominent features. ${ }^{[9]}$ Galbusera et al. ${ }^{[9]}$ in 2006 described revised diagnostic criteria for TTP in which the diagnosis can be made upon identification of the presence of thrombocytopenia and microangiopathic haemolytic anaemia (MAHA) only. Other atypical manifestations of TTP that have been described include non-occlusive mesenteric ischaemia, pancreatitis, hepatitis, acute respiratory distress syndrome and peripheral digital ischaemia. ${ }^{[1,11]}$

TTP is a severe illness and, if untreated, $>90 \%$ of patients die from the syndrome. ${ }^{[12]}$ Plasmapheresis is the treatment of choice for TTP, in addition to steroids, which are initiated immediately after start of plasma exchange therapy (PEX). ${ }^{[8]}$ Rituximab is effective and safe in acute idiopathic TTP and in patients with immune TTP who have failed to respond to PEX and steroids. ${ }^{[13]}$ The poor outcome of untreated patients is a big challenge particularly in SSA, where there are poor health systems and poor access to quality healthcare.

We report a case of a 46-year-old man who presented with most of the classic clinical manifestations TTP.

\section{Case presentation}

A 46-year-old male patient born deaf and dumb presented to the Aga Khan University Hospital as a referral from a peripheral facility, with a 2-week history of severe headache, abdominal pains, diarrhoea and vomiting. He had been fine prior to the onset of symptoms and there was no history of travel to a malaria endemic region. The headache was occipital, of sudden onset, severe (scale 8 - 10/10), nonradiating, worsened with activity and was temporarily relieved by use of analgesics. There was no associated fever or neck stiffness. He had visual hallucinations with no other visual symptoms. Diarrhoea was watery and not blood stained, and the vomiting was mostly postprandial with associated epigastric abdominal pain. There was no history of decreased urine output, lower-limb swelling and facial swelling, and no respiratory symptoms. His past medical history and family history were non-significant. He worked as a tailor and was married with three children. Physical examination showed a well-developed, middle-aged black African male patient of good nutritional status and with stable vital signs. He had mild pallor, was not jaundiced, had no lymphadenopathy and no oedema, and skin examination was normal. He communicated by sign language, his neck was supple and the rest of the neurological examination findings were unremarkable. Abdominal, respiratory and cardiovascular examinations were unremarkable. Laboratory findings showed severe anaemia (haemoglobin $5.8 \mathrm{mg} / \mathrm{dL}$ ), thrombocytopenia (platelet counts $\left.9 \times 10^{9}\right)$, increased lactate dehydrogenase activity (517 IU/L) and schistocyte count was $3 \%$. Renal and liver function tests were normal with a negative direct Coombs test. HIV antibody, hepatitis B surface antigen and hepatitis $C$ virus antibody tests were negative. Blood and stool cultures were negative, while renal and liver function tests were normal; it is important to note that he had been on intravenous antibiotics in the referring facility. His blood slide for malaria parasites was also negative. 
TTP was suspected because of the haemolytic anaemia, thrombocytopenia, schistocytes on the peripheral blood film and the neurological manifestations. The patient was started on PEX at a dose of one volume PEX daily, and the efficacy of the plasma exchange was monitored using daily thrombocyte levels. Despite being on daily PEX, his thrombocytes did not improve until after 5 days of therapy. After 1 week in the hospital, undergoing PEX, he was noted to have declining mental status, and increased aggressiveness and disorientation. Magnetic resonance imaging was not done because of the need for sedation. Computed tomography scan of the brain showed small bilateral subdural haematomas with no pressure effects; the patient was put on conservative management with continued monitoring of neurological status. He improved and there was no need for neurosurgical intervention.

After seven sessions of PEX, the thrombocytes improved to $157 \times 10^{9} / \mathrm{L}$. PEX was stopped after the thrombocyte levels remained above $150 \times 10^{9} / \mathrm{L}$ for 2 consecutive days. Three days after PEX had been stopped, the thrombocytes dropped to $47 \times$ $10^{9} / \mathrm{L}$, and a diagnosis of refractory TTP was made. Financial constraints limited subsequent PEX to one more session, and only one dose of rituximab (375 $\mathrm{mg} / \mathrm{m}^{2}$ body weight) for refractory TTP was given. The thrombocytes improved and the patient's clinical condition improved tremendously, and he was discharged 4 days after the dose of rituximab. The thrombocyte count improved to $160 \times 10^{9} / \mathrm{L}$ on starting rituximab and to $198 \times 10^{9} / \mathrm{L}$ on review in a clinic 2 weeks later. The patient was discharged on prednisolone, tapered over 3 months. He was doing very well 3 months post discharge, with no symptoms, and he had returned to work. He had a platelet count of $253 \times 10^{9} / \mathrm{L}$ and $233 \times 10^{9} / \mathrm{L}$ on review 2 and 3 months post discharge, respectively (Fig. 1).

\section{Discussion}

TTP was diagnosed in this patient owing to the presence of thrombocytopenia, MAHA with schistocytes of $3 \%$ and neurological manifestations. ${ }^{[9]}$ Systemic bacterial and fungal infections were excluded with several negative blood cultures, as severe sepsis is one of the differentials for schistocytosis and thrombocytopenia. ${ }^{[14]}$ Patients with severe sepsis may also present with disseminated intravascular coagulation with a subsequent lack of procoagulant proteins, causing bleeding diathesis and anaemia.

TTP is a life-threatening condition, especially in SSA, where optimal treatment facilities are not available. PEX was initiated owing to a presumptive diagnosis of TTP because of the associated high mortality of up to $90 \%$ in untreated patients. ${ }^{[12]} \mathrm{PEX}$ is the mainstay of treatment in TTP as it reduces mortality from $90 \%$ to $10-20 \%{ }^{[8]}$ This modality of treatment depletes the circulating antibodies against ADAMTS13 and the very high molecular weight von Willebrand factor multimers. PEX also replaces the missing von Willebrand cleaving protease. ${ }^{[15]}$ Although the mortality of TTP has been reduced in most European countries and in America, we postulate that TTP still has a high mortality in SSA owing to lack of PEX. Management of refractory TTP is a bigger challenge owing to the need for more prolonged duration of treatment. However, more intensive PEX may be required in resistant cases with new-onset symptoms while on therapy ${ }^{[16]}$ Our patient improved tremendously but deteriorated 2 days postPEX, with thrombocytes dropping to 47 . Owing to financial constraints, only one additional session of PEX was done for refractory TTP and one dose of rituximab was given. Fakhouri et al. ${ }^{[13]}$ showed that rituximab is effective and safe in immune TTP for patients who have failed PEX and

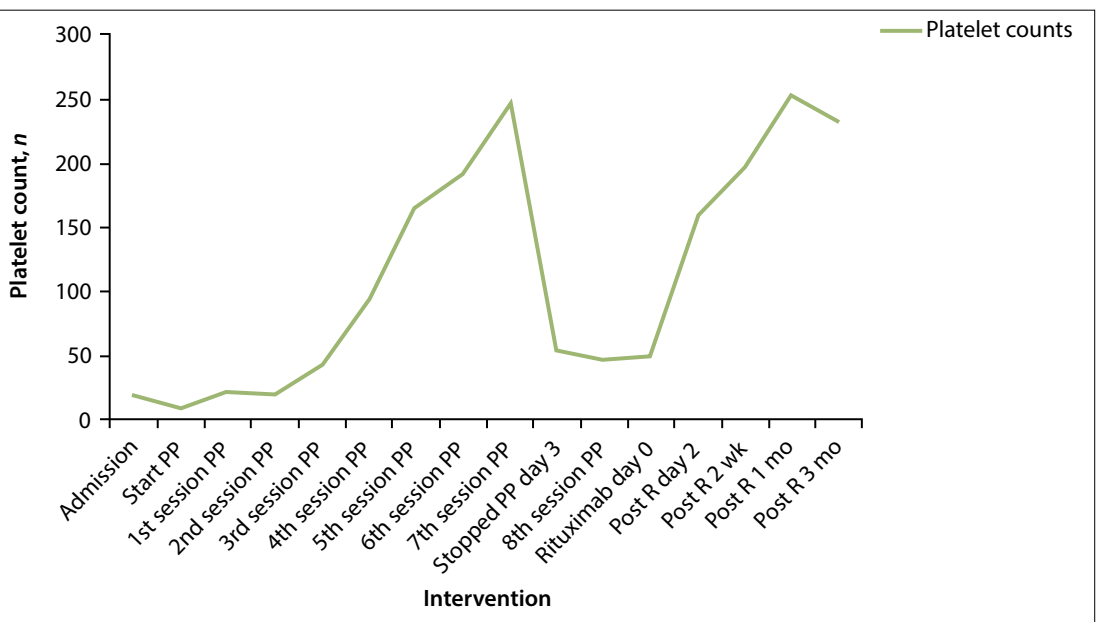

Fig. 1. Platelet counts against intervention. $(R=$ rituximab; $w k=$ weeks; $m o=$ months. $)$ methylprednisone treatment and in acute relapsed TTP. Rituximab is the current treatment of choice for refractory TTP and should be given once weekly for 4 weeks; ${ }^{[3,8]}$ our patient was started on rituximab early because of the challenges of PEX in our setting. Rituximab in combination with prednisone is effective and can be used without concurrent plasma exchange in patients without neurological or renal impairment. ${ }^{[17]}$ Long-term consequences of TTP are not well known, but it has been postulated that some patients may go on to develop longterm renal dysfunction and neurocognitive deficits. $^{[15]}$

This case report demonstrates both the possibilities and challenges of managing TTP. Diagnosis and initiation of appropriate therapy according to international guidelines was possible for this patient and a relatively good response was demonstrated. However, our patient did not receive optimal care owing to financial constraints; this is a common scenario in SSA. Our patient responded well to one dose of rituximab. Further studies are necessary to explore the viability of use of rituximab alone in settings where PEX is not possible.

\section{Conclusions}

TTP can present without the full pentad of the classic clinical presentation. There should be increased suspicion of TTP in patients who present with MAHA, especially in a population that has a high prevalence of HIV infection. This is the first reported case of TTP that was relatively appropriately managed with plasma exchange and rituximab in this part of the developing world. Rituximab has been used for patients with resistant and relapsed TTP with successful results in other regions of the world; these results can be replicated in our setting. Further studies to assess the feasibility and viability of the use of rituximab alone in settings where PEX is not possible are necessary.

1. Fujikawa K, Suzuki H, McMullen B, Chung D. Purification of human von Willebrand factor-cleaving protease and its identification as a new member of the metalloproteinase family. Blood 2001;98(6):1662-1666. DOI:10.1182/blood. v98.6.1662

2. Maoke I Thrombotic microangiopathies. N Engl J Med 2002;347(8):589-600. DOI:10.1056/NEJMra020528

3. Scully M, Yarranton H, Liesner R, et al. Regional UK TTP registry: Correlation with laboratory ADAMTS13 analysis and registry: Correlation with laboratory ADAMTS13 analysis and
clinical features. Br J Haem 2008;142(5):819-826. DOI:10.1111/ clinical features. Br $)$ Hae

j.1365-2141.2008.07276.

4. Furlan M, Robles R, Galbusera M, et al. Von Willebrand factor-cleaving protease in thrombotic thrombocytopenic purpura and the hemolytic-uremic syndrome. N Engl J Med 1998;339(22):1578-1584. DOI:10.1056/NEJM199811263392202

5. Bitzan M, Schaefer F, Reymond D. Treatment of typical (enteropathic) hemolytic uremic syndrome. Semin Thromb Hemost 2010;36(6):594-610. DOI:10.1055/s-0030-1262881

6. Novitzky N, Thomson J, Abrahams L, du Toit C, McDonald A. Thrombotic thrombocytopenic purpura in patients with retroviral infection is highly responsive to plasm infusion therapy Br J Haem 2005;128(3):373-379. DOI:10.1111/j.1365-2141.2004.05325.x 
7. Gunther K, Garizio D, Nesara P. ADAMTS13 activity and the presence of acquired inhibitors in human immunodeficiency virus-related thrombotic thrombocytopenic purpura. Transfusion 2007:47(9):1710-1716. DOI:10.1111/j.1537-2995.2007.01346.

8. Scully M, Hunt BJ, Benjamin S, et al. Guidelines on the diagnosis and management of thrombotic thrombocytopenic purpura and other thrombotic microangiopathies. Br J Haem 2012:158(3):323-335. DOI:10.1111/j.1365-2141.2012.09167.x

9. Galbusera M, Noris M, Remuzzi G. Thrombotic thrombocytopenic purpura - then and now. Semin Thromb Hemost 2006;32(2):81-89. DOI:10.1055/s-2006-939763

10. Chang JC, Gupta S. Acute respiratory distress syndrome and non-occlusive mesenteric ischemia as major clinical manifestations of thrombotic thrombocytopenic purpura: Complete remission following exchange plasmapheresis. J Clin Apher 1998;13(4):190-192.

11. Chang JC, Kathula SK. Various clinical manifestations in patients with thrombotic microangiopathy. J Investig Med 2002;50(3):201-206. DOI:10.2310/6650.2002.33434

12. Patschan D, Korsten P, Behlau A, et al. Idiopathic combined, autoantibody-mediated ADAMTS-13/ factor $\mathrm{H}$ deficiency in thrombotic thrombocytopenic purpura-hemolytic uremic syndrome in 17-year-old woman: A case report. J Med Case Rep 2011;5(1):598. DOI:10.1186/1752-1947-5-598
13. Fakhouri F, Vernant JP, Veyradier A, et al. Efficiency of curative and prophylactic treatment with rituximab in ADAMTS13-deficient thrombotic thrombocytopenic purpura: A study of 11 cases. Blood 2005;106(6):1932-1937. DOI:10.1182/blood-2005-03-0848

14. Irmak K, Sen I, Cöl R, et al. The evaluation of coagulation profiles in calves with suspected septic shock 4. Irmak K, Sen I, Cöl R, et al. The evaluation of coagulation profiles in calve
Vet Res Commun 2006;30(5):497-503. DOI:10.1007/s11259-006-3258-8

15. See JR, Sabagh T, Barde CJ. Thrombotic thrombocytopenic purpura: A case presenting with acute See JR, Sabagh T, Barde CJ. Thrombotic thrombocytopenic purpura: A case preser
ischemic colitis. Case Rep Hematol 2013;2013:592930. DOI:10.1155/2013/592930

16. Nguyen L, Li X, Duvall D, Terrell DR, Vesely SK, George JN. Twice-daily plasma exchange for patients with refractory thrombotic thrombocytopenic purpura: The experience of the Oklahoma Registry 1989 through 2006. Transfusion 2008;48(2):349-357. DOI:10.1111/j.1537-2995.2007.01530.x

17. Ahmad A, Aggarwal A, Sharma D, et al. Rituximab for treatment of refractory/relapsing thrombotic thrombocytopenic purpura (TTP). Am J Hematol 2004;77(2):171-176. DOI:10.1002/ajh.20166

Accepted 17 March 2016. 